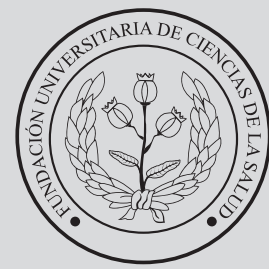

FUCS
Re T

\title{
Medidas de frecuencia por COVID-19 en Bogotá D.C.
}

\section{Measures of frequency of COVID-19 in Bogota D.C.}

a Ingeniero. Magister en Gestión de la Tecnología Educativa, Especialista en Administración de la Informática Educativa. Docente de matemáticas e Investigador, Secretaría de Educación de Soacha, Cundinamarca.

\section{R E S U M E N}

Introducción: A medida que el virus COVID-19 continúa contagiando a personas en todo el mundo, hay poco conocimiento de las implicaciones a largo plazo para los pacientes recuperados. Hay informes de síntomas constantes después de infecciones confirmadas en pacientes, incluso después de tres meses de la recuperación inicial. Objetivo: estimar las medidas de frecuencias de prevalencia, mortalidad y letalidad en las veinte localidades de Bogotá para COVID-19. Metodología: Para desarrollar la investigación se utilizó la base de datos de las personas contagiadas y fallecidas por COVID-19, la información de los datos correspondiente al período acumulado a 20 de agosto de 2020 para Bogotá. Resultados: Se determinó que la localidad con mayor prevalencia fue Sumapaz con $28,47 \%$, la localidad con mayor tasa de mortalidad fue Tunjuelito con una tasa de 0,9 y la localidad con mayor porcentaje de letalidad por cada 100 habitantes está también en Tunjuelito con una tasa de 3,6. Conclusiones: se ha evidenciado en lo corrido de 2020 un aumento paulatino de contagios por COVID-19 en la ciudad de Bogotá, hasta llegar a registrar a 21 de agosto de 2020, 179.540 casos positivos, con un porcentaje a nivel nacional de 34,95\%. La tendencia de casos positivos, mortalidad y letalidad, seguramente seguirá aumentando hasta que no se encuentre una solución definitiva a la pandemia propiciada por este virus.

Palabras clave: COVID-19, pandemia, mortalidad, letalidad, prevalencia.

(C) 2020 Fundación Universitaria de Ciencias de la Salud - FUCS Este es un artículo Open Access bajo la licencia CC BY-NC-ND (http://creativecommons.org/licenses/by-nc-nd/4.0/).

\section{INFORMACIÓN DEL ARTÍCULO}

Historia del artículo:

Fecha recibido: agosto 31 de 2020

Fecha aceptado: septiembre 4 de 2020
Autor para correspondencia. Ing. Jorge Enrique Díaz Pinzón jediazp@unal.edu.co
DOI

10.31260/RepertMedCir.01217372.1110 
Introduction: As the COVID-19 virus continues to infect people around the world, much is still unknown about the longterm effects in recovered patients. There are reports of confirmed COVID-19 patients who experience lingering symptoms even three months after their initial recovery. Objective: to estimate the measures of frequency of prevalence, mortality and lethality of COVID-19 in the twenty localities of Bogota. Methodology: The database including people who were infected and have died from COVID-19 disease was used for the research, with the information accumulated for Bogota up to August 20 2020. Results: It was determined that the locality with the highest prevalence was Sumapaz with $28.47 \%$, the locality with the highest mortality rate, was Tunjuelito with a rate of 0.9 and the locality with the highest percentage of lethality per 100 inhabitants, was also Tunjuelito, with a rate of 3.6. Conclusions: There has been a gradual increase in COVID-19 infections in the city of Bogotá throughout 2020. A total of 179.540 positive cases were registered as to August 21 2020, accounting for $34.95 \%$ of the national case tally. The trend of positive cases, mortality and lethality, will steadily rise until a definitive solution to the pandemic caused by this virus is found.

Key words: COVID-19, pandemic, mortality, lethality, prevalence.

(C) 2020 Fundación Universitaria de Ciencias de la Salud - FUCS. This is an open access article under the CC BY-NC-ND license (http://creativecommons.org/licenses/by-nc-nd/4.0/).

\section{INTRODUCCIÓN}

A medida que el virus COVID-19 continúa contagiando a personas en todo el mundo, hay poco cocimiento de las implicaciones a largo plazo para los pacientes recuperados. Ha habido informes de síntomas constantes después de infecciones confirmadas en pacientes, incluso después de tres meses de la recuperación inicial. ${ }^{1}$

El diagnóstico clínico de COVID-19 bosqueja un enorme desafío para la detección y prevención tempranas que es de crucial grado para la contención de pandemias. ${ }^{2}$ La enfermedad hoy representa una advertencia mundial para la salud pública. ${ }^{3}$ La nueva pandemia mundial de la enfermedad por coronavirus 2019 (COVID-19) ha sido una carga importante para los hospitales y los proveedores de atención médica. ${ }^{4}$

Los resultados revelan que las medidas de cierre tienen efectos reveladores para alentar a las personas a mantener el distanciamiento social. Sin embargo, la gravedad de la pandemia y los factores socioeconómicos e institucionales tienen efectos condicionados para mantener el distanciamiento. Los resultados también exponen que los factores socioeconómicos e institucionales de la urbanidad y la modernidad tienen efectos reveladores sobre la gravedad de la pandemia. ${ }^{5}$

El distanciamiento social es una medida central de salud pública en el combate contra la pandemia de COVID-19, pero la obediencia de los individuos no puede darse por sentado. ${ }^{6}$ A medida que el mundo se reabre, los riesgos de nuevos brotes están creciendo ${ }^{7}$, esto debido a la velocidad de transmisión y el número de reproducción básico. ${ }^{8}$ Por lo tanto, la enfermedad del coronavirus 2019 (COVID-19), con una tasa de mortalidad en ancianos (60 años o más) mucho más alta que los pacientes jóvenes (menores de 60 años), fue declarada pandemia por la Organización Mundial de la Salud el 11 de marzo de 2020 teniendo en cuenta esta tasa de mortalidad dependiente de la edad. ${ }^{9}$

En el caso de Colombia, según Díaz ${ }^{10}$, en su trabajo de investigación "Descripción estadística del COVID- 19 según el grupo etario en Colombia" entre el 6 y 10 de julio de 2020, determinó que los grupos etarios con mayor promedio de personas fallecidas por COVID-19 fueron: $70-79$ (1.120,6); 60-69 (1.060,0); 80-89 (883,2); 50-59 $(645,6) ; 40-49(347,4) ;$ y los grupos con menor promedio de recuperados fueron: $90-99(249,8)$; 30-39 (156,0); 20-29 $(67,4) ; 0-9(11,0) ; y$ 10-19 $(6,0)$.

Ahora, el objetivo de la investigación es calcular la proporción, la razón de contagio y recuperación de personas por COVID-19. Además de hallar el porcentaje de mortalidad y letalidad en las 20 localidades de Bogotá, con la información proveniente de Salud Capital ${ }^{11}$ con fecha de corte 20 de agosto 2020 (tabla 1). Para llevar a cabo este propósito, en epidemiología frecuentemente se trabaja con diferentes tipos de fracciones que admiten cuantificar el impacto de una explícita enfermedad. ${ }^{12-14}$

\section{La prevalencia (P)}

La prevalencia cuantifica la proporción de individuos de una población que padecen una enfermedad en un momento o periodo de tiempo determinado. ${ }^{14-16} \mathrm{Su}$ cálculo se estima mediante: 


\section{Número de casos con la enfermedad en un momento dado \\ Prevalencia $=$ \\ Total de población en ese momento}

"Como todas las proporciones, la prevalencia no tiene dimensión y nunca toma valores menores de 0 a mayores de 1 , siendo frecuente expresarla en términos de porcentaje, en tanto por ciento, tanto por mil, en función de la "rareza" de la enfermedad estudiada". ${ }^{15}$

\section{Mortalidad general}

"La mortalidad general es el volumen de muertes ocurridas por todas las causas de enfermedad, en todos los grupos de edad y para ambos sexos. La mortalidad general, que comúnmente se expresa en forma de tasa, puede ser cruda o ajustada, de acuerdo con el tratamiento estadístico que reciba. La tasa cruda de mortalidad se estimó mediante"12,14.

\begin{tabular}{|c|c|}
\hline Tasa de & $\begin{array}{l}\text { Número de muertes en un } \\
\text { periodo determinado }\end{array}$ \\
\hline general & $\begin{array}{l}\text { Población total promedio en el } \\
\text { mismo periodo }\end{array}$ \\
\hline
\end{tabular}

\section{Tasa de letalidad}

La letalidad es una medida de la gravedad de una enfermedad estimada desde el punto de vista poblacional, y se precisa como la proporción de casos de una enfermedad que surgen mortales con respecto al total de casos en un periodo determinado. La medida muestra la relevancia de la enfermedad en términos de su disposición para producir la muerte y se deduce de la siguiente manera ${ }^{12,14}$.

Letalidad $(\%)=\frac{\begin{array}{c}\text { Número de muertes por una } \\ \text { enfermedad en un periodo } \\ \text { determinado }\end{array}}{\begin{array}{c}\text { Número de casos diagnosticados } \\ \text { de la misma enfermedad en el } \\ \text { mismo periodo }\end{array}} \times 100$

La letalidad, en sentido exacto, es una proporción ya que enuncia el número de defunciones entre el número de casos del cual las defunciones forman parte. Sin embargo, universalmente se expresa como tasa de letalidad y se registra como el porcentaje de muertes de una causa específica con respecto al total de enfermos por esa causa. ${ }^{12,14}$

\section{RESULTADOS}

\section{Cálculo de la prevalencia por contagio por COVID-19}

Para el cálculo de la prevalencia por contagio por COVID-19, se tuvo en cuenta la población por localidades en Bogotá con la información acumulada a 20 de agosto de 2020, según la información registrada en Saludata como se aprecia en la tabla 1.

Tabla 1. Casos positivos por COVID-19 y total de la población por localidades Bogotá a 20 de agosto de 2020

\begin{tabular}{|c|c|c|}
\hline Localidad & $\begin{array}{c}\text { Total de la } \\
\text { población }\end{array}$ & $\begin{array}{c}\text { Casos } \\
\text { positivos }\end{array}$ \\
\hline Usaquén & 501999 & 8950 \\
Chapinero & 139701 & 3621 \\
Santa Fe & 110048 & 3403 \\
San Cristóbal & 404697 & 8839 \\
Usme & 457302 & 7641 \\
\hline Tunjuelito & 199430 & 4950 \\
Bosa & 673077 & 15255 \\
Kennedy & 1088443 & 24017 \\
Fontibón & 394648 & 7223 \\
Engativá & 887080 & 15540 \\
Suba & 1218513 & 21624 \\
Barrios Unidos & 243465 & 3128 \\
Teusaquillo & 153025 & 2629 \\
Los Mártires & 99119 & 2651 \\
Antonio Nariño & 109176 & 2479 \\
Puente Aranda & 258287 & 6463 \\
La Candelaria & 24088 & 727 \\
Rafael Uribe Uribe & 374246 & 9910 \\
Ciudad Bolívar & 707569 & 13279 \\
Sumapaz & 6531 & 1860 \\
Total & $\mathbf{8 ' 0 5 0 . 4 4 4}$ & $\mathbf{1 7 9 5 4 0}$ \\
\hline
\end{tabular}

Fuente. Elaboración propia.

En la figura 1 se aprecian los datos de la prevalencia en porcentaje (\%), para cada una de las veinte localidades de Bogotá, se detalla que las localidades con mayor prevalencia de casos positivos por COVID-19, están: Sumapaz con 28,47\%, Santa Fe con 3,1\%, La Candelaria 3,01\%, Los Mártires 2,67\%, y Rafael Uribe Uribe con un 2,64\%; y las localidades con menor proporción tenemos: Barrios Unidos 1,28\%, Usme 1,6\%, Teusaquillo 1,7\% y Engativá 1,7\%, a nivel Bogotá 2,23\%.

\section{Cálculo de la tasa de mortalidad específica (TME) por COVID-19}

En la figura 2 se determinaron las tasas de mortalidad especificas (TME) por COVID-19, por localidades en Bogotá, se aprecia que la localidad con mayor TME por cada 1000 habitantes está en primer lugar Tunjuelito con una tasa de 0,9, Rafael Uribe Uribe 0,76, Puente Aranda 0,7, San Cristóbal 0,68, Kennedy 0,6, Ciudad Bolívar 0,56, Bosa 0,52, Engativá y Usme 0,5, Fontibón 0,47, Suba 0,46, Usaquén 0,41, y las localidades de Barrios Unidos, Teusaquillo, Antonio Nariño, Chapinero, Los Mártires, La Candelaria, Santa Fe y Sumapaz con 0,0. El total a nivel de Bogotá 0,5, es decir que en todas las localidades y en Bogotá, no alcanza a fallecer una persona por cada 1000 habitantes. 


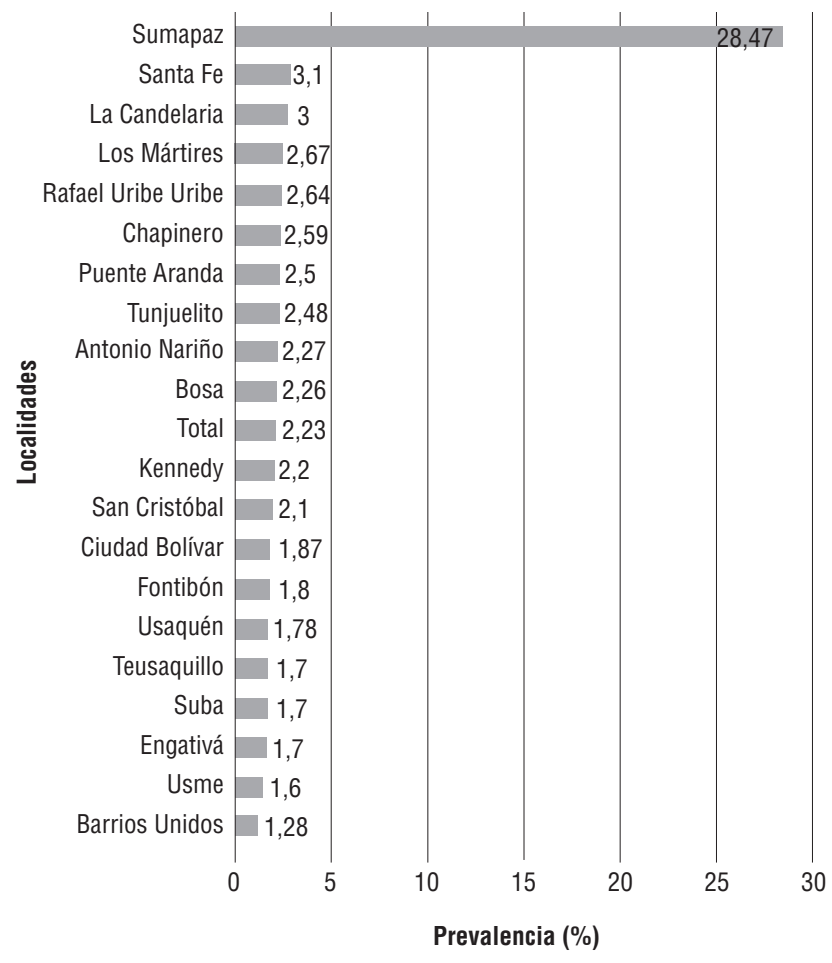

Fuente. El autor

Figura 1. Prevalencia de contagio por COVID-19 por localidades en Bogotá, a 20 de agosto 2020.

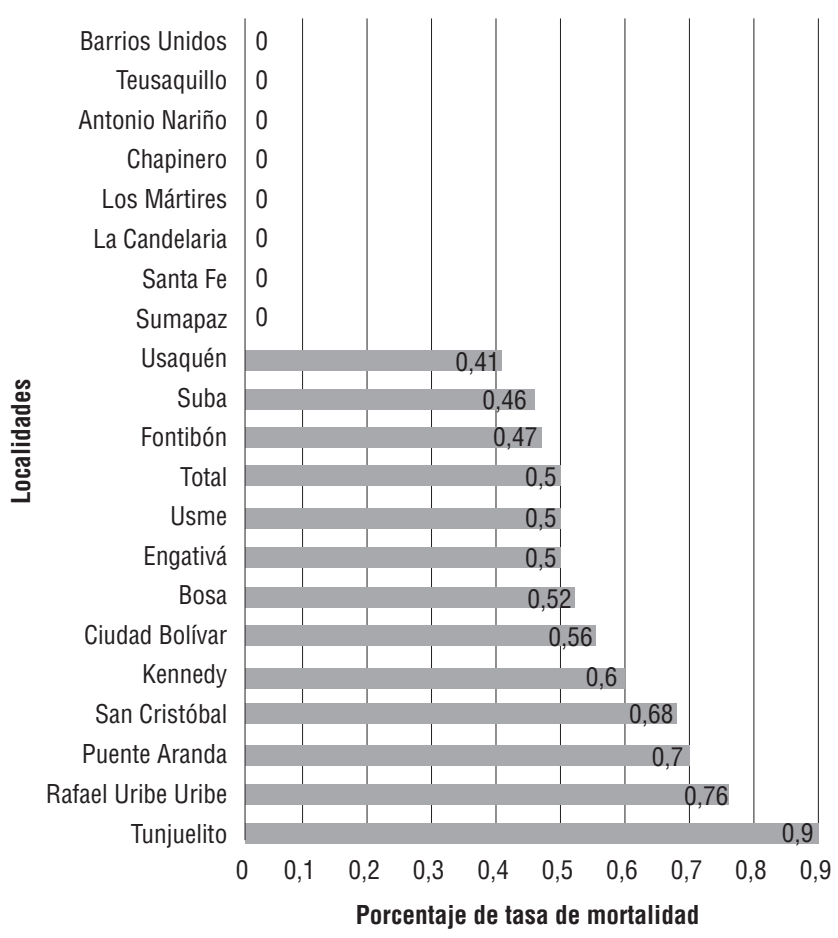

Fuente. El autor

Figura 2. Tasas de mortalidad específica por COVID-19 por localidades en Bogotá a 20 de agosto 2020.

\section{Cálculo de la tasa de letalidad por COVID-19}

En la figura 3 se estableció las tasas de letalidad por COVID-19, por localidades en Bogotá, se aprecia que la localidad con mayor porcentaje de letalidad por cada 100 habitantes está en primer lugar Tunjuelito con una tasa de 3,6, San Cristóbal 3,12, Usme y Ciudad Bolívar 3,0; es decir que 3 de cada 100 casos positivos fallece. Seguimos con Engativá 2,9, Rafael Uribe Uribe 2,88, Puente Aranda 2,76, Kennedy 2,72, Suba 2,63, Fontibón 2,58, Usaquén 2,32, Bosa 2,32; es decir que 2 de cada 100 casos positivos fallece. En las localidades de Barrios Unidos, Teusaquillo, Antonio Nariño, Chapinero, Los Mártires, La Candelaria, Santa Fe, Sumapaz con 0,0; no se presentan fallecimientos de las personas contagiadas, y el total a nivel Bogotá es de 2,21, es decir que 2 de cada 100 casos positivos fallece.

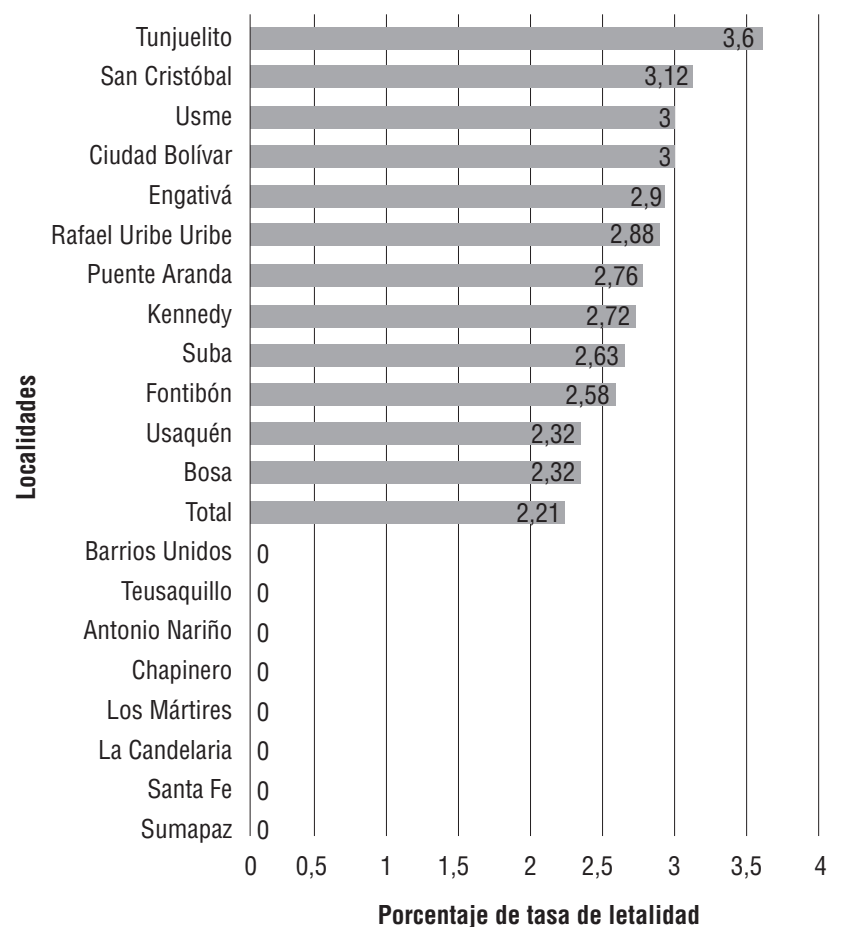

Fuente. El autor

Figura 3. Tasas de letalidad por COVID-19 por localidades en Bogotá a 20 de agosto 2020.

\section{CONCLUSIÓN}

Se estimó al 20 de agosto 2020, la prevalencia para cada una de las veinte localidades de Bogotá; se detalla que las localidades con mayor prevalencia de casos positivos por COVID-19, son: Sumapaz con un 28,47\%, Santa Fe 3,1\%, La Candelaria 3,01\%, Los Mártires 2,67\%, y Rafael Uribe Uribe $2,64 \%$. Las localidades con menor proporción tenemos: Barrios Unidos con un 1,28\%, Usme 1,67\%, Teusaquillo $1,71 \%$ y Engativá 1,75\%, a nivel Bogotá 2,23\%. 
Se determinaron las tasas de mortalidad especificas (TME) por COVID-19, por localidades en Bogotá; se aprecia que la localidad con mayor TME por cada 1000 habitantes está en primer lugar Tunjuelito con una tasa de 0,9, Rafael Uribe Uribe 0,76, Puente Aranda 0,7, San Cristóbal 0,68, Kennedy 0,6, Ciudad Bolívar 0,56, Bosa 0,52, Engativá y Usme 0,5, Fontibón 0,47, Suba 0,46, Usaquén 0,41, y las localidades de Barrios Unidos, Teusaquillo, Antonio Nariño, Chapinero, Los Mártires, La Candelaria, Santa Fe, Sumapaz con 0,0; y el total a nivel Bogotá 0,5 , es decir que en todas las localidades y en Bogotá, no alcanza a fallecer una persona por cada 1000 habitantes.

Esto comparado con Díaz ${ }^{17}$ en su trabajo de investigación "Estimación de las tasas de mortalidad y letalidad por COVID-19 en Colombia" donde determinó que, el grupo etario que presentó mayor tasa de mortalidad es el de 90-99, con 338 muertes por cada 100.000 habitantes, seguido del grupo entre 80-89, con 335 muertes por cada 100 habitantes, y los grupos con menor tasa de mortalidad de 0-9 y 10-19, que no alcanzan a tener una sola muerte por cada 100.000 habitantes. A nivel nacional la tasa por cada 100.000 habitantes fue 26 muertes.

Ahora, las tasas de letalidad por COVID-19, por localidades en Bogotá, se aprecia que la localidad con mayor porcentaje de letalidad por cada 100 habitantes está en primer lugar Tunjuelito con una tasa de 3,6, San Cristóbal 3,12, Usme y Ciudad Bolívar 3,0; es decir que 3 de cada 100 casos positivos fallece. Seguimos con Engativá 2,9, Rafael Uribe Uribe 2,88, Puente Aranda 2,76, Kennedy 2,72, Suba 2,63, Fontibón 2,58, Usaquén 2,32, Bosa 2,32; es decir que 2 de cada 100 casos positivos fallece. En las localidades de Barrios Unidos, Teusaquillo, Antonio Nariño, Chapinero, Los Mártires, La Candelaria, Santa Fe, Sumapaz con 0,0; no se presentan fallecimientos de las personas contagiadas y el total a nivel Bogotá es de 2,21, es decir que 2 de cada 100 casos positivos fallece.

\section{REFERENCIAS}

1. Banda JM, Singh GV, Alser O, Prieto-Alhambra D. Long-term patient-reported symptoms of COVID-19: an analysis of social media data. medRxiv. 2020:2020.07.29.20164418. DOI: https:// doi.org/10.1101/2020.07.29.20164418

2. Karni N, Klein H, Asseo K, Benjamini $\mathrm{Y}$, Israel S, Nimri $M$, et al. Self-rated smell ability enables highly specific predictors of COVID-19 status: a case control study in Israel. medRxiv. 2020:2020.07.30.20164327. DOI: https://doi. org/10.1101/2020.07.30.20164327

3. Utamura M, Koizumi M, Kirikami S. Isolation Considered Epidemiological Model for the Prediction of COVID-19 Trend in Tokyo, Japan. medRxiv. 2020:2020.07.31.20165829. DOI: https:// doi.org/10.1101/2020.07.31.20165829

4. Tsai A, Diawara O, Nahass RG, Brunetti L. Impact of tocilizumab administration on mortality in severe COVID-19. medRxiv.
2020:2020.07.30.20114959.

DOI: https://doi.org/10.1101/2020.07.30.20114959

5. Rahman MM, Thill J-C, Paul KC. COVID-19 Pandemic Severity, Lockdown Regimes, and People Mobility: Evidence from 88 Countries. medRxiv. 2020:2020.07.30.20165290.

DOI: https://doi.org/10.1101/2020.07.30.20165290

6. Becher M, Stegmueller D, Brouard S, Kerrouche E. Comparative experimental evidence on compliance with social distancing during the COVID-19 pandemic. medRxiv. 2020:2020.07.29.20164806. DOI: https://doi.org/10.1101/2020.07.29.20164806

7. Freitag MO, Schmude J, Siebenschuh C, Stolovitzky G, Hamann H, Lu S. Critical Mobility, a practical criterion and early indicator for regional COVID-19 resurgence. medRxiv. 2020:2020.07.30.20163790. DOI: https://doi. org/10.1101/2020.07.30.20163790

8. Petford N, Campbell J. Covid-19 mortality rates in Northamptonshire UK: initial sub-regional comparisons and provisional SEIR model of disease spread. medRxiv. 2020:2020.07.30.20165399. DOI: https://doi.org/10.1101/2020.07.30.20165399

9. Yang HM, Lombardi LP, Morato Castro FF, Yang AC. Mathematical modeling of the transmission of SARS-CoV-2 $\otimes$ Evaluating the impact of isolation in São Paulo State (Brazil) and lockdown in Spain associated with protective measures on the epidemic of covid-19. medRxiv. 2020:2020.07.30.20165191. DOI: https://doi. org/10.1101/2020.07.30.20165191

10. Díaz Pinzón JE. Descripción estadística del COVID-19 según el grupo etario en Colombia. Repert Med Cir. 2020;29(Supl. Núm.1):79-85. DOI: https://doi.org/10.31260/RepertMedCir.01217372.1098

11. Salud Capital. Datos de salud enfermedades transmisibles. Casos confirmados de COVID-19 en Bogotá D.C. [Internet]. Bogotá: Saludata, observatório de Salud de Bogotá; 2020 [citado 2020 agoso 21]; Disponible en: http://saludata.saludcapital.gov.co/osb/ index.php/datos-de-salud/enfermedades-trasmisibles/covid19/.

12. Moreno-Altamirano A, López-Moreno MC, Sergio , CorchoBerdugo A. Principales medidas en epidemiología. Salud Pública de México. 2000;42(4):337-48.

13. Instituto Nacional de Estadística e Informática (INEI). Metodología para el Cálculo de losIndicadores de Mortalidad. Metodologías Estadísticas. 2000;1(8):1-9.

14. Quintana-Salgado L. Medidas de frecuencia en epidemiología [Internet]. 2015 [citado 2020 julio 14]; Disponible en: https:// es.slideshare.net/lualberts20/medidas-de-frecuencia-enepidemiologa-2015.

15. Pita Fernández S, Pértegas Díaz S, Valdés Cañedo F. Medidas de frecuencia de enfermedad [Internet]. España: Elsevier; 2004 [citado 2020 julio 14]; Disponible en: https://www.fisterra.com/formacion/ metodologia-investigacion/medidas-frecuencia-enfermedad/.

16. Pinto A. Prevalencia e Incidencia [Internet]. Slideshare; 2014 [cited 2020 agosto 21]; Available from: https://es.slideshare.net/ alexpinto18/prevalencia-e-incidencia-2.

17. Díaz Pinzón JE. Estimación de las tasas de mortalidad y letalidad por COVID-19 en Colombia. Repert Med Cir. 2020;29(Núm Supl.1):8993. DOI: https://doi.org/10.31260/RepertMedCir.01217372.1103 